\title{
A CASE Tool Platform Using an XML Representation of Java Source Code
}

\author{
Katsuhisa Maruyama ${ }^{\dagger}$ \\ Department of Computer Science \\ Ritsumeikan University \\ 1-1-1 Noji-higashi Kusatsu \\ Shiga 525-8577, Japan \\ maru@cs.ritsumei.ac.jp
}

\author{
Shinichiro Yamamoto \\ Department of Information Systems \\ Aichi Prefectural University \\ 1522-3 Ibaragabasama Kumabari Nagakute-cho \\ Aichi-gun Aichi 480-1198, Japan \\ yamamoto@ist.aichi-pu.ac.jp
}

\begin{abstract}
Recent IDEs have become more extensible tool platforms but do not concern themselves with how other tools running on them collaborate with each other. They compel developers to use proprietary representations or the classical abstract syntax tree (AST) to build source code tools. Although these representations contain sufficient information, they are neither portable nor extensible. This paper proposes a tool platform that manages commonly used, finedgrained, information about Java source code by using an $X M L$ representation. Our representation is suitable for developing tools which browse and manipulate actual source code since the original code is annotated with tags based on its structure and retained within the tags. Additionally, it exposes information resulting from global semantic analysis, which is never provided by the typical AST. Our proposed platform allows the developers to extend the representation for the purpose of sharing or exchanging various kinds of information about the source code, and also enables them to build new tools by using existing XML utilities.
\end{abstract}

\section{Introduction}

Object-oriented software is hard to develop without integrated development environments (IDEs) since it consists of many classes and contains the relationship between them. A significant point is that a recently released IDE is not only a collection of programming tools but also an extensible tool platform. For example, Eclipse [3] has a powerful plug-in mechanism for easily adding new tools to itself and removing existing tools from itself.

By supporting the plug-in mechanism, developers have a chance to build their own tools and would want their tools

\footnotetext{
$\dagger$ Visitor at the Institute for Software Research (ISR), University of California, Irvine, from September 2003 through September 2004.
}

to collaborate with each other. Accordingly, a tool platform must collect the detailed information about tools (or programs) being developed and then present it in proper form that can meet developers' diverse requirements. Unfortunately, conventional tool platforms store information about source code by using either proprietary representations or the typical abstract syntax tree (AST) [14]. Of course these representations contain sufficient information and several powerful tool platforms such as Eclipse [3], the DMS Software Reengineering Toolkit [10], or RECORDER [9] provide well-designed application programming interfaces (APIs) for accessing the information.

However, the classical representations are neither portable nor extensible. That is, none of the conventional platforms concern themselves with how a newly built tool stores the additional information obtained through its execution and exchanges such information with other tools. In addition, the proprietary or fundamental APIs are insufficient for building diverse tools. Therefore, the tool developers tend to create overhead modules, which are used for extracting necessary information from the integrated representation, in their respective tools, or might have to modify the integrated modules and date structure. To build various kinds of software tools managing source code and make them collaboratively work together without too much effort, the tool platform should not only use a simple standard but also portable and extensible representation. Such a representation would act as a medium for exchanging sourcecode information and would allow the developers to add individual information they define.

The authors have developed a tool platform with a software repository that can store and provide fine-grained information about Java source code by using the extensible markup language (XML) [4]. This paper proposes this tool platform and a new XML-based representation, which are called Sapid/XML (sophisticated APIs for CASE tool de- 
velopment with an XML repository) and XSDML (extensible software document markup language), respectively. XSDML documents are converted from source code; code fragments are classified by marking them with respective tags and are structured by nesting the tags based on the structure of the source code. Additionally, these documents contain additional information resulting from syntactic and semantic analysis.

Sapid/XML provides a fine-grained XML representation which is an alternative to the classical AST of Java source code. It makes the source code more portable and convenient since XSDML exposes the structure and relationship found in the source code and the format is based on XML. XML is a simple, widely used text-based format that is used to design markup languages suitable for the capture and exchange of information. Many existing XML utilities can be used for examining and manipulating the source code. Sapid/XML also allows developers to extend the prepared representation although its extension would need a simple consistency check for the document type definition (DTD) [4]. They can define new tags and attributes to share common information and exchange specific information. It is useful for building new software tools to extend the prepared representation of source code without examining and modifying modules in the tool platform. Here the authors have to mention that Sapid/XML does not strive to dismiss existing IDEs. It shows the potential of a tool platform using an XML representation of the source code.

We first describe existing XML representations of object-oriented source code. Next we present an overview of Sapid/XML and explain how Java source code is converted to an XSDML document and how software tools access the converted XSDML document. Then we show several software tools running on Sapid/XML and give experimental results in respect to the performance of Sapid/XML. Finally, we conclude with a summary.

\section{XML Representations of Source Code}

Several XML formats currently exists for representing object-oriented source code. For example, GraX [19] and GXL [23] are classified into a graph-based format. They store information about nodes and edges of the abstract syntax graph (ASG) [6] without reflecting the nested structure of the source code in its XML representation. On the other hand, Harmonia [17], JavaML [15], OOML (cppML and JavaML) [27], bison-based parser [29], XMLizer [28], FreeTXL [18], and srcML [26, 25] directly encode actual source code or its AST to the nested structure of their XML representations. These representations are all intended to exchange information about source code or display the abstract structure of the source code. They are fulfilling such purpose since XML is a simple, extensible, widely used text-based format. This concept is analogous to that of our XSDML. However, the main purpose of Sapid/XML is to facilitate developers in building tools which manipulate actual source code.

From this point of view, XSDML (and Sapid/XML) is closely related to srcML although their target programming languages are different (Java and $\mathrm{C}++$, respectively). All of the conventional representations except srcML never support the representation of comments or formatting [26]. That is, only srcML and XSDML preserve the original text of source code containing formatting information and guarantee the restoration of the complete original source code. Moreover, both of them have several common features: tag names based on programmers' knowledge (i.e., syntactic names such as classes, methods, or fields) and the conversion that directly inserts tags to source code as meta-data.

While XSDML is similar to srcML, there are three main differences between them. First, XSDML provides all fragments of source text (operators or separators, identifiers, keywords, white spaces, and new lines) with dedicated tags (see Section 3.1). These tags allow developers or tools to add extra white spaces and new lines that were not contained in the original source code. The original white spaces and new lines are always enclosed with terminal elements while extra ones are enclosed with non-terminal elements. Secondly, XSDML aggressively exploits many kinds of attributes while very few attributes are used in srcML (see Section 3.1). The verbose attributes alleviate additional lexical analysis of the contents of elements or the timeconsuming traversal of several elements when the developers and tools obtain the properties of code fragments (e.g., modifiers). Finally, XSDML contains several useful links obtained through global (and local) semantic analysis for the whole of source code (Section 3.2). Some of the links are used in GXL or JavaML but are not provided by srcML.

\section{Sapid/XML Tool Platform}

Sapid/XML generates the XML documents represented in our proposed XSDML from Java programs (written in Java 1.4 or earlier) and provides them for software tools. Figure 1 shows an overview of the Sapid/XML tool platform. It mainly consists of four components: a source code converter (a syntactic parser and a semantic analyzer), access libraries, a Java-XML software repository, and Java wrappers. This section explains how Java programs are converted into XSDML documents and what information is contained in these documents, and describes the access libraries and the Java wrappers accessing the documents.

\subsection{Syntactic Parser}

XSDML represents the classical text-based source code as 20 non-terminal elements and 7 terminal ones, which are 


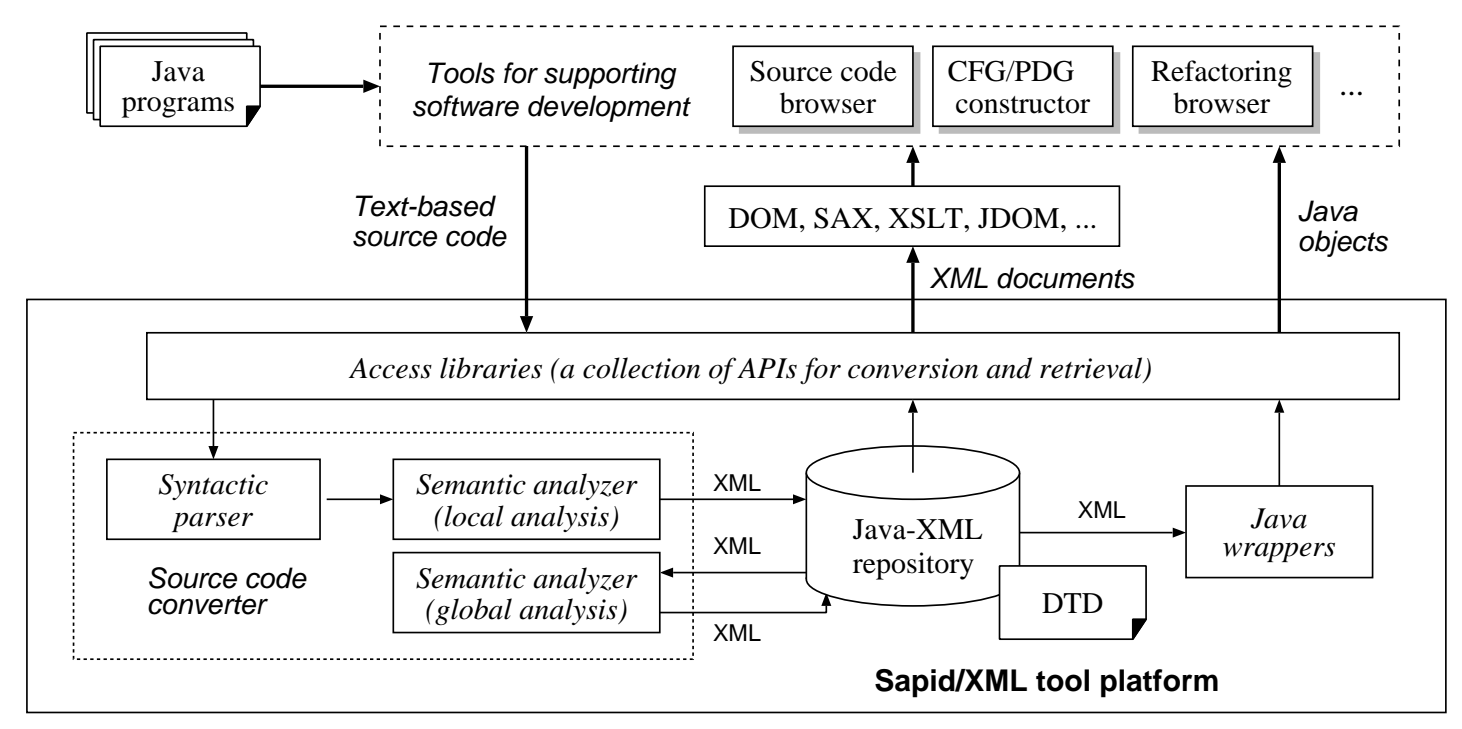

Figure 1. Overview of the proposed tool platform.

shown in Table 1. The terminal element has only the textual contents while the non-terminal element can nest others. The syntactic parser directly inserts these elements into the original source code without changing the contents of the code, that is, it only adds tags and attributes in the original code. Each of the code fragments is delimited by the tags and all the tokens (identifiers, keywords, comments, white spaces, and new lines) of the code remain in the textual contents of the terminal elements. The original source code can be restored from the converted XSDML document by removing all the tags and leaving behind the textual contents of the terminal elements. The attributes are available to represent additional properties such as modifiers, accessibility settings, fully-qualified names, and sorts of elements. For example, the type (Type), statement (Stmt), expression (Expr), and literal (I iteral) elements are classified as $3,15,59$, and 6 by the attribute sort, respectively ${ }^{1}$.

The simple source code quoted from [15] and the XSDML document converted from it are shown in Figures 2 and 3, respectively. Each line of the document except the XML headers corresponds to that of the source code since the XSDML retains every new line character. Moreover, the original code can be seen in the textual contents of the terminal elements (e.g., the blanks or keywords are enclosed with the $<\mathrm{sp}>$ or $<\mathrm{kw}>$ ). This crude document is hard for a human to read but we can use various XML utilities to view it. Figure 4 illustrates the tree view of the XSDML document shown in Figure 3 as displayed by the Mozilla [8].

Here it is worth discussing the problems Badros pointed out in [15]. He stated that the representation marked-up by only adding tags would need further lexical analysis of the

\footnotetext{
${ }^{1}$ See http://www.jtool.org for details.
}

Table 1. Elements of XSDML

\begin{tabular}{|l|l|}
\hline Element name & Fragment of Java source code \\
\hline File & Compilation unit (File) \\
Package & Package declaration \\
Import & Import declaration \\
Class & Class declaration \\
Intf & Interface declaration \\
SInit & Static initializer \\
Ctor & Constructor declaration \\
Method & Method declaration \\
Field & Field declaration \\
Param & Formal parameter \\
Local & Local variable declaration \\
Extdopt & Superclass clause \\
Implopt & Superinterface clause \\
ThrwOpt & Throws clause \\
Members & Class/interface body \\
Qname & Qualified identifier \\
Type & Type \\
Stmt & Statement \\
Label & Label declaration \\
Expr & Expression \\
\hline ident & Identifier \\
literal & Literal \\
comment & Comment \\
kw & Keyword \\
op & Operator \\
sp & Blank or tab character \\
nl & New line character \\
\hline
\end{tabular}

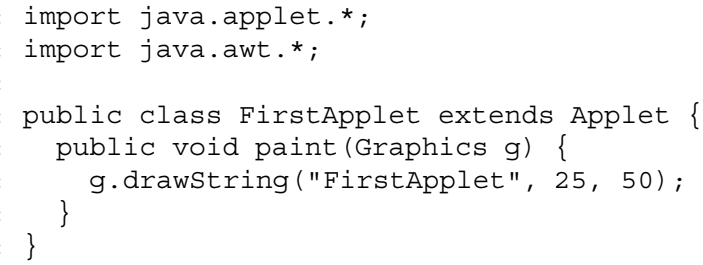

Figure 2. Original Java source code. 
$<$ ?xml version="1.0"?>

$<$ !DOCTYPE File SYSTEM "JX-model3-ext.dtd">

<File classpath="/usr/home/maru/Work/Report/scam04/xsdml-examples/FirstApplet" id="s792723457" pat. .

$</$ nl $><$ Import id="do" $><k w>$ import $</ \mathrm{kw}><$ sp $></$ sp $><$ QName id="s843055105" $><$ ident defid="s843055105" $>$ jav. .

$</ \mathrm{nl}><\mathrm{nl}$ line="3" offset $=" 41 ">$

$</ \mathrm{nl}><$ Class access="Public" fqn="FirstApplet" id="s796917761" $><\mathrm{kw}>$ public $</ \mathrm{kw}><\mathrm{sp}></ \mathrm{sp}><\mathrm{kw}>\mathrm{Class}</$.

$</ \mathrm{nl}><\mathrm{sp}>\quad</ \mathrm{sp}><$ Method access="Public" id="s809500673" typefirst="s813694978" $><\mathrm{kw}>$ public $</ \mathrm{kw}><$ sp $>$. .

$</ \mathrm{nl}><\mathrm{sp}>\quad</ \mathrm{sp}><$ Stmt id="s826277890" sort="EXPR" $><$ Expr id="s830472193" sort="DOT" $><$ Expr id="s83.

$</ \mathrm{nl}><\mathrm{sp}>\quad</ \mathrm{sp}><$ op $>\}</$ op $></$ Stmt $></$ Method $><$ nl line="7" offset="162" >

$</$ nl $><$ op $>\}</$ op $></$ Members $><$ Ances distance="0" name="FirstApplet" sort="CLASS" $></$ Ances $><$ Ances distan. .

$</$ nl $><$ FqnMap fqn="java. awt. MenuContainer" jar="rt.jar" path="java/awt/MenuContainer.class" $></$ FqnMa. .

Figure 3. Document represented in XSDML.

textual contents, and it would not sufficiently abstract the original source code. To alleviate these problems, XSDML introduces a fine-grained tagging and slightly verbose attributes. For example, the method call at line 6 in the source code shown in Figure 2 is converted into ${ }^{1}$ :

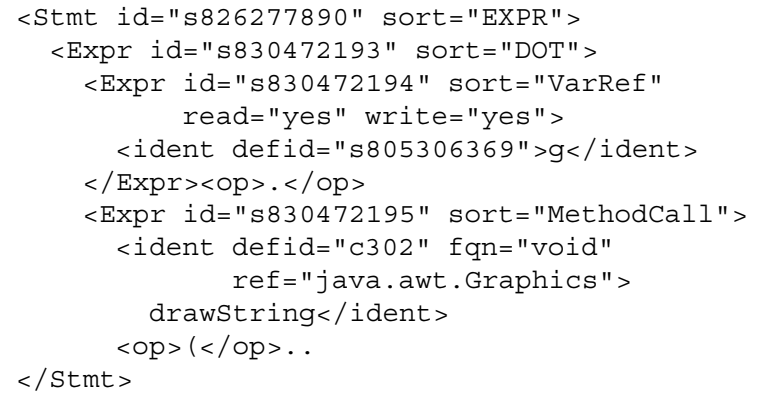

The statement (Stmt) corresponding to the method call is decomposed into some detailed elements. Another example for the method declaration from line 5 through 7 is as follows:

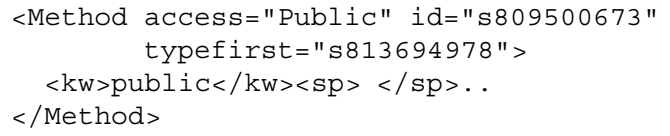

The value of the access attribute and the textual contents in $\langle\mathrm{kw}\rangle$ are redundant. As shown in the above two examples, the XSDML representation no longer requires lexical analysis although it retains the contents of the original code. Moreover, these examples indicate that our conversion is suitable for implementing tools which manipulate actual source code and browse it without changing its appearance since white spaces (tabs and blanks) and new lines remain. For example, a refactoring browser or a code checker works well with our representation since most tool users do not want it to remove comments or formatting characters (white spaces and new lines) from the original source code. The highly abstract representation such as JavaML is insufficient to implement these software tools although its representation would be convenient for making a survey of

\footnotetext{
${ }^{1}$ Blanks and new lines are inserted in the examples presented hereafter so that the readers can easily read them.
}

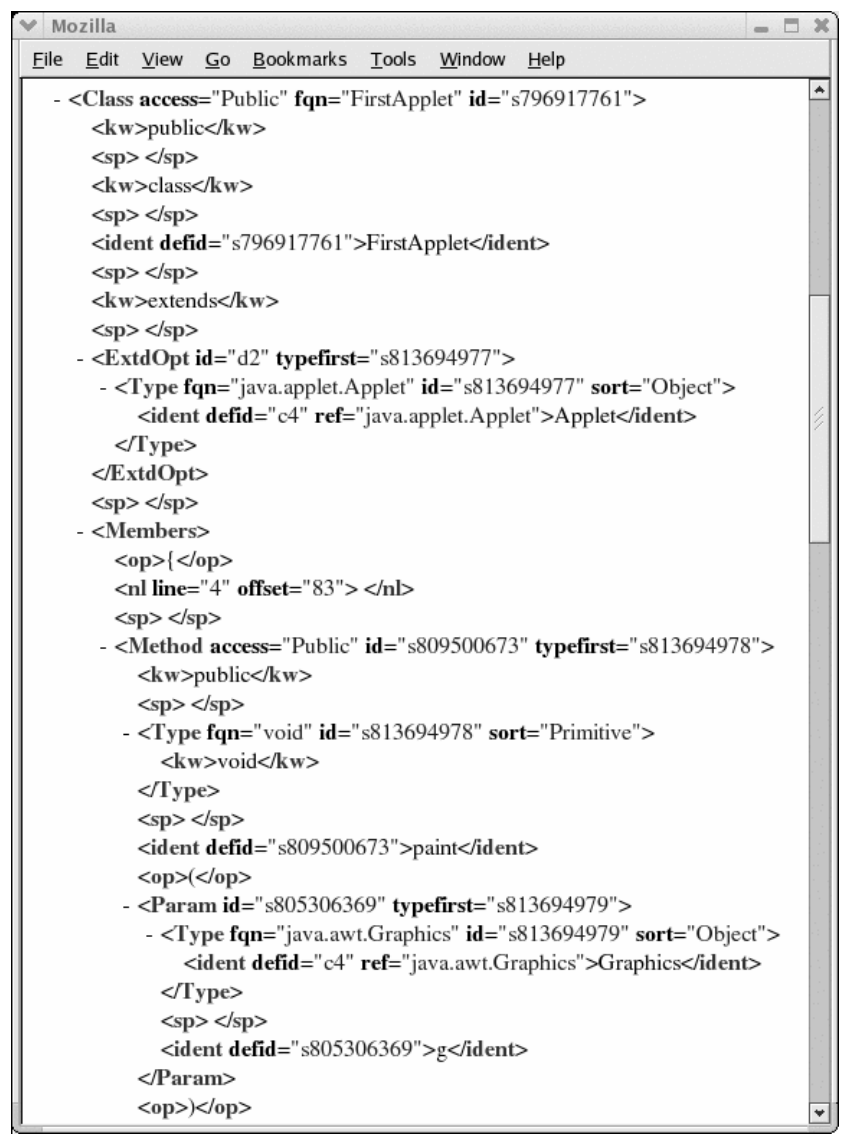

Figure 4. Tree view of the XSDML document.

the source code or measuring its metrics and some tools requiring such a representation independent to a specific programming language.

\subsection{Semantic Analyzer}

The significant feature of Sapid/XML is that it reflects information based on semantic analysis in its XML representation. The semantic analyzer inserts two kinds of information: type and reference. The type information is expressed by the fqn attribute. For the type Graphics at line 
5 in the source code shown in Figure 2, the following description is generated.

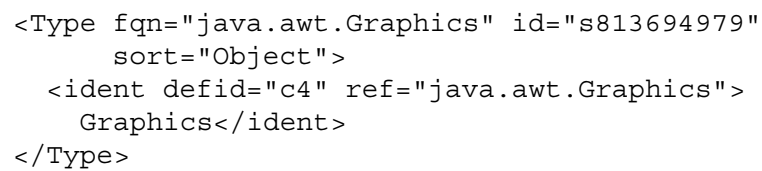

It can be easily seen that the fully-qualified name of Graphics is java.awt.Graphics because of the value of fqn. The fully-qualified name is determined based on the search path for class and jar files, and used for obtaining the next reference information.

The reference information is classified as a local or global link. The local link is expressed by both the $i d$ and defid attributes like the JavaML. The defid indicates the link of the call or access to the element the $i d$ value of which equals to the defid value. A referenced element is always decided since the id value must be unique within an XSDML document. XSDML enhances this notation to express global links across several XML documents by adding the ref attribute. For example, the following description:

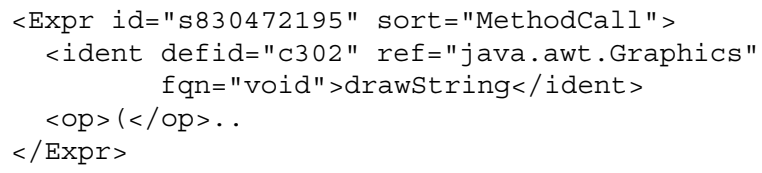

indicates invocation to the method drawString the id value of which equals to $\mathrm{c} 302$ in the class java.awt.Graphics. The $f q n$ attribute denotes the return type. The link of the field access is represented in the same manner.

Along with the reference information, the read and/or write attributes are added to all Expr elements corresponding to the references to fields and local variables. For example, the variable $\mathrm{g}$ which is a primary expression of the method call to drawString is represented as follows:

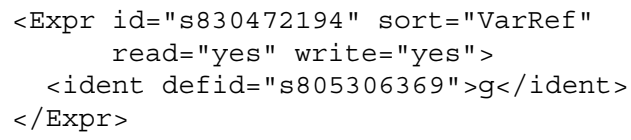

The read= "yes" or write= "yes" means that the variable is used without or with changing its value, respectively. The write="yes" is added when the state of the object indicated by the reference variable might change (i.e., the value of any fields defined in the object might be written).

The process of determining which method would be called and which field would be accessed is similar to that done when compiling source code (Section 15.11 and 15.12 in [21]). It is based on the apparent (or declarative) type of a related object since an actual object is decided at run-time and its precise type is not known at compiletime. The apparent type is obtained from the value of the fqn attribute corresponding to the primary ident or Expr element. Here the careful readers will wonder why
java.awt.Graphics has the id attribute. Sapid/XML uses the byte code engineering library (BCEL) [1] and automatically generates summary XML documents from class (and jar) files whenever the files are referred by the analyzed class. Moreover, it determines which classes should be reanalyzed when a class is changed, by utilizing the global link information (and specially adding the new tags Ances and FqnMap). If any ancestor of the specified class, any class it refers to, or itself is modified, the platform automatically re-generates a new XSDML document from it.

The type and reference (plus read/write) information is often extracted by existing tools but is not reusable in general. For example, most compilers lose part of the information after generating final class files. Although some of them store the information in the class files, its format is hard to read because of optimization. Sapid/XML makes such information more explicit and provides it in an easyto-use format in order that software tools easily query and manipulate source code. This is significant since such semantic analyzer is expensive to build from scratch. Moreover, the provided link information must be common and fundamental to all kinds of software tools although it is not enough to build them without supplemental information.

\subsection{Access Libraries and Wrappers}

Every XSDML document is stored in the Java-XML repository. Tools running on Sapid/XML can request access to the libraries to convert Java programs into XSDML documents or to retrieve some of them from the repository with several queries. The retrieved documents can be used through various XML utilities, (e.g., the document object model (DOM) [2], the simple API for XML (SAX) [11], the extensible stylesheet language (XSL) and XSL transformations (XSLT) [5], and JDOM [7]). For example, the following Java code using DOM APIs outputs the name of all methods existing in a Java source file of interest.

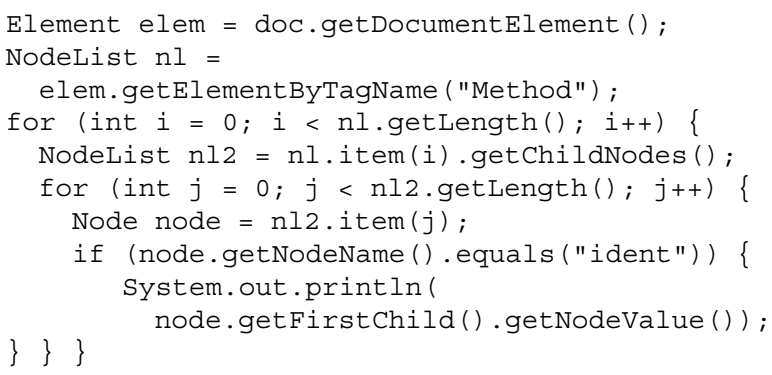

The doc variable indicates a document object of the XSDML document generated from the source file.

The standard APIs (e.g., DOM and SAX) are of course convenient for writing code independent to a specific programming language but too primitive for most developers when they build tools in practice. Accordingly, the developers tend to write tedious code repeatedly. To avoid this rep- 


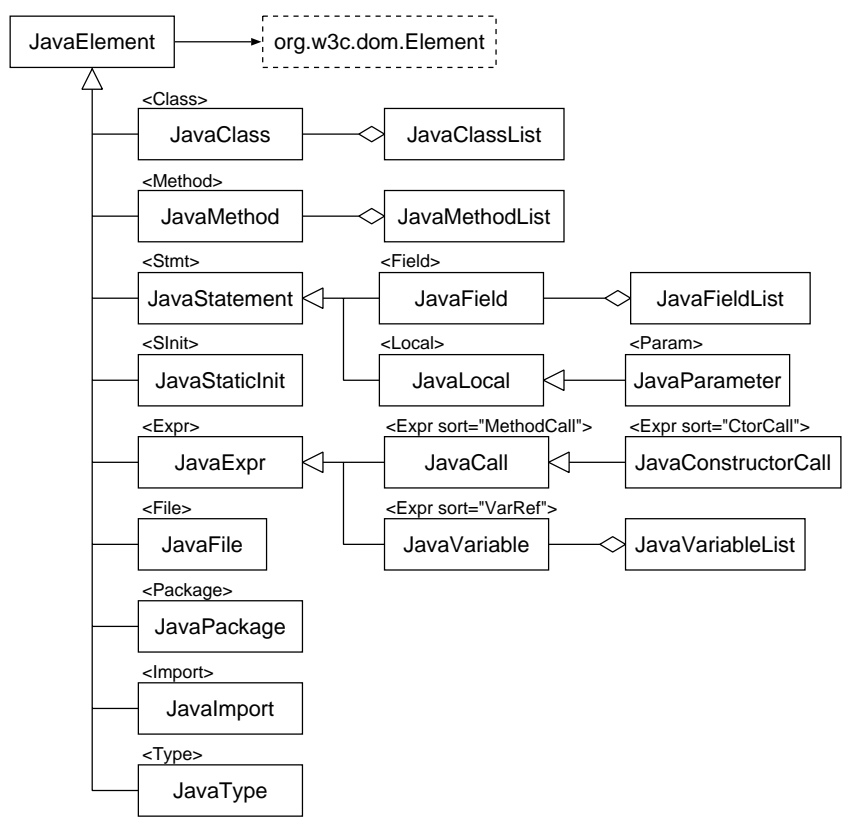

Figure 5. Java wrappers for XSDML.

etition, Sapid/XML provides several Java wrappers which have high-level APIs for accessing XSDML documents. In Figure 5, the rectangles denote the wrappers corresponding to the XSDML elements depicted in the top of them.

The wrappers are classes tool developers would frequently use and allow them to easily access portions of a DOM tree in the Java object form. For example, the code getting a list of classes in the Java source file (indicated by doc) is as follows:

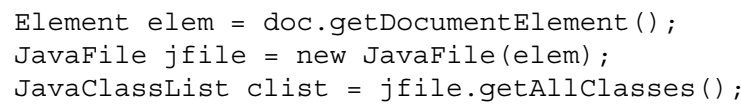

In addition, the code outputting the name of all methods existing in a class is as follows:

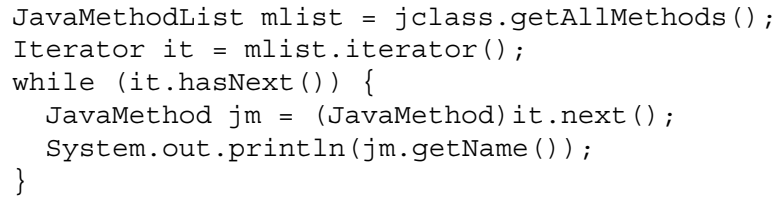

The variable jclass is an object of the JavaClass wrapper. All wrappers are designed only to extract information from XSDML documents and never change their contents. They are also useful samples of writing code that accesses and manipulates the XSDML documents.

\section{Practical Tools Using Sapid/XML}

One strength of Sapid/XML is that it structures Java source code with several tags and embeds additional information resulting from semantic analysis in the converted
XML documents. By specifying tags in querying and transformation, portions of the code can be accessed and extracted. Moreover, Sapid/XML neither loses tokens of original source code nor adds superfluous texts to the textual contents of terminal elements when generating XSDML documents. This feature is convenient for modifying only the part of source code and retaining the remaining code, or marking (or highlighting) source code without changing its appearance. Most source code viewers and editors do not desire tool platforms to arbitrarily change the contents of source code (e.g., indentations or the position of braces) since they have their individual formatters.

To evaluate these benefits, we have developed the following tools.

- A method viewer generating a HTML document listing the declaration of methods for each class.

- A source code browser generating a browsable code containing hyperlinked references in HTML form.

- A CFG/PDG constructor producing a control flow graph (CFG) [14] and a program dependence graph (PDG) [20] for each method existing in source code.

- A cross-reference extractor collecting link information about inverse references (e.g., callers of a method) and relationships (e.g., method override), and producing XML documents containing the information.

- A refactoring browser restructuring existing source code without changing its observable behavior.

Due to space limitation, we will explain only the former three tools in this paper. ${ }^{2}$

\subsection{Method Viewer}

The method viewer is a simple XSLT application. Figure 6 shows a web browser displaying method declarations in source code. It was trivial to identify classes, methods, and constructors since they were marked with Class, Method, and Ctor in the converted XSDML document, respectively. Carefully looking at Figure 6, all class (or type) names in the method declarations were replaced with fully-qualified ones. Displaying such information is easily performed by using the value of the fqn attribute of Type elements instead of their actual names. With Sapid/XML, tools can obtain various kinds of information about source code through XML utilities and thus developers can build such tools without writing much code.

\subsection{Source Code Browser}

The source code browser is also an XSLT application. The stylesheet is described in Appendix A. Figure 7 shows

\footnotetext{
${ }^{2} \mathrm{All}$ of these tools can be downloaded from http://www.jtool.org.
} 


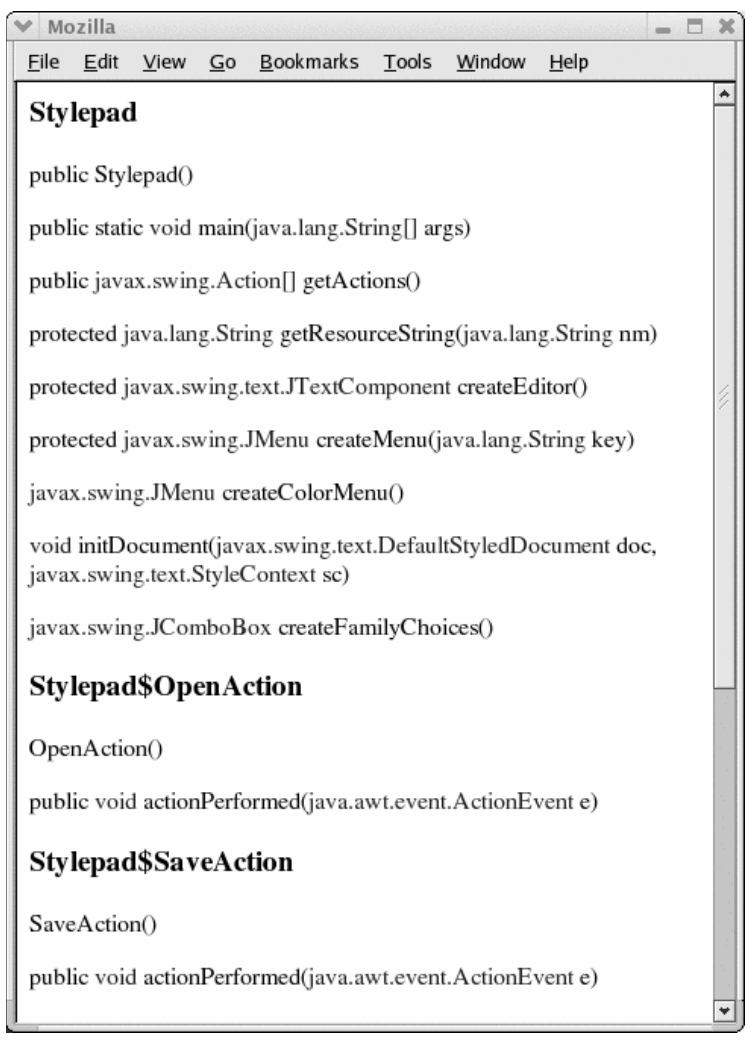

Figure 6. Viewing the declaration of methods.

a view of the generated HTML-based source code. This stylesheet performs mainly two transformations. One is to enclose the name (ident) of classes, methods, fields, local variables with the $<$ a name $="\{@$ defid $\} ">$ and $\langle/ a\rangle$ elements. The @defid indicates the value of the defid attribute of elements owning the sandwiched names.

The other transformation is to find references to classes, methods, fields, and local variables, and enclose the references with $<$ a href $="\{$ \$relpath $\}\{$ path $\} . h t m l$ \#\{@defid\}" > and </a > elements. As mentioned in Section 3.2, all references in XSDML documents have the defid attribute, the value of which indicates the target element and substitutes for @defid. Moreover, global references (other than references to local variables) have the ref attribute which indicates the fully-qualified name of a class containing the target element. The \$path is obtained through the FqnMap map storing the correspondences between the fully-qualified name of a class and the name of a file containing the class. The \$relpath denotes a relative path to the top of directories storing HTML files and is provided as a parameter of the stylesheet.

Tags except for newly added ones are removed and the textual contents of all elements are left behind. A significant point is that the appearance of the restored source code is the exactly same as that of the original source code.

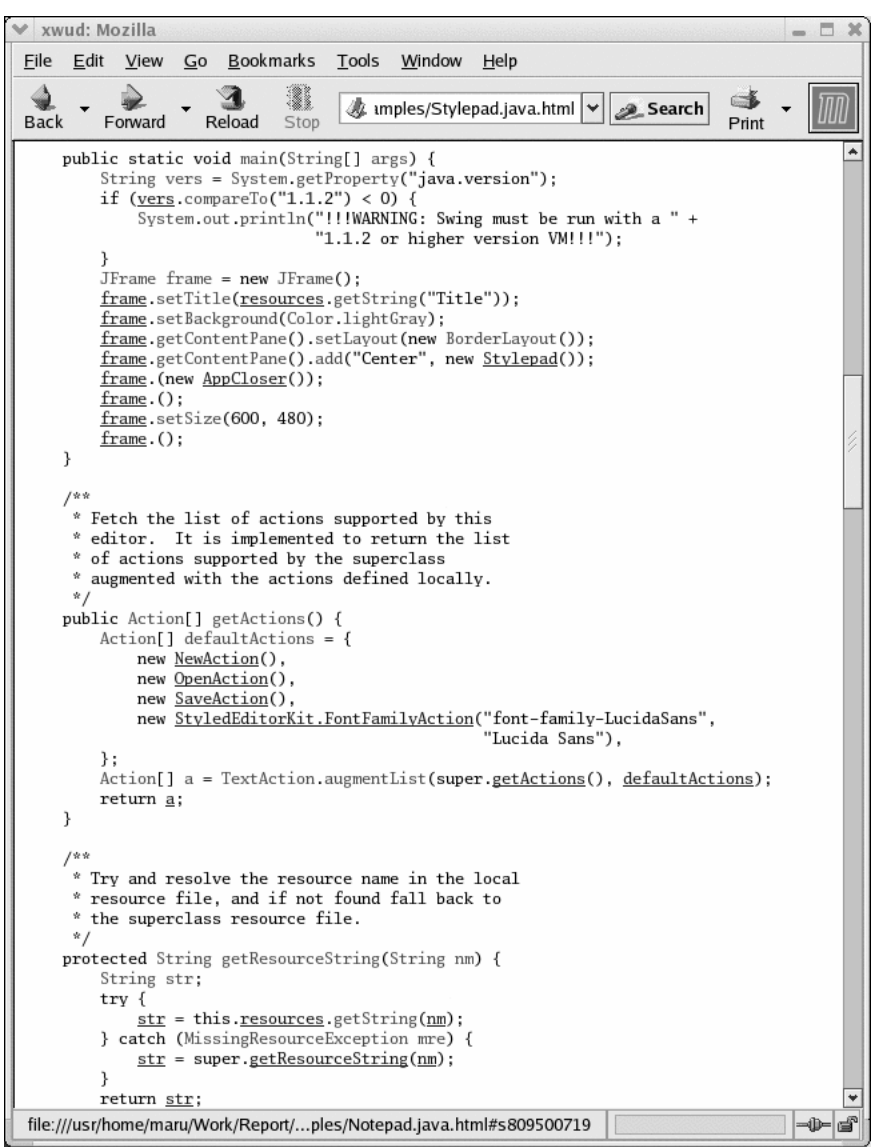

Figure 7. Viewing HTML-based source code.

Sapid/XML is well suited for creating this kind of tool because it preserves all tokens of the original source code in converted XSDML documents.

\subsection{CFG/PDG Constructor}

The CFG and PDG (or control and data flow) are often used for creating tools that support software development. For example, the CFG is useful for eliminating dead code or code clone, and the PDG is invaluable for debugging or testing. Program slicing [30] is a famous application using the PDG, which is widely applied to various fields. Our developed refactoring browser uses this CFG/PDG constructor. The information about CFGs and PDGs can be obtained through both XML documents and Java objects.

The CFG consists of a set of nodes and edges. Each node denotes a statement which is either an assignment or a condition predicate, which is marked the Stmt or Expr tag. Each edge represents immediate control flow from a statement and another one. An example of the generated CFG is as follows:

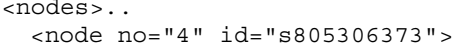




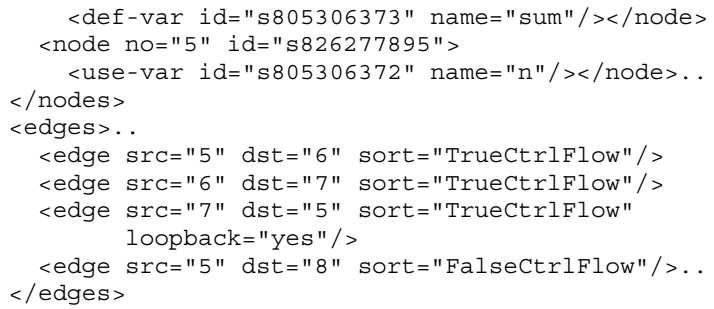

The src or dst attribute denotes the value of the no attribute of a source or destination node, respectively. The sort attribute is either Truectrlflow (if-then), FalseCtrlflow (if-else), or FallThrFlow [16]. The loopback="yes" means its edge is a back-edge for a loop. The analyzer of the current version of Sapid/XML cannot deal with control flow involved in exception. To alleviate this problem, a path edge [22] which indicates control flow for exception handling will be embedded.

Similar to the CFG, the PDG consists of a set of nodes and edges. Each node corresponds to a node of the CFG generated from the same source code. Edges denote control and data dependences. A control dependence edge represents a control condition on which the execution of a statement depends. Data dependence edge represents flow of data between statements, which is classified as either loopcarried or loop-independent [24]. An example of the generated PDG is as follows:

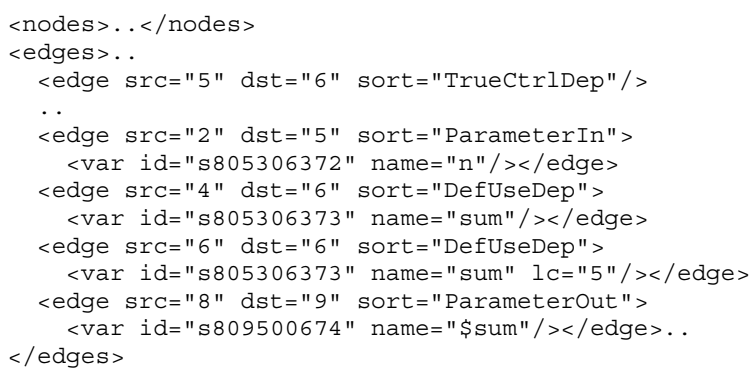

The sort attribute equals either TrueCtrlDep (true control dependence), FalsectrlFlow (false control dependence), DefUseDep (def-use data dependence), ParameterIn (def-use data dependence related to an incoming parameter), or ParameterOut (def-use data dependence related to an outgoing parameter). The $1 \mathrm{c}$ attribute in a var element indicates a loop-node carrying the edge enclosing the var element.

In general, the dependency analysis of the whole program is too expensive. Consequently, the current CFG/PDG constructor does not further analyze the variable appearing in the primary expression of method invocation or field access. Such a variable is considered to be modified and thus the created PDGs are all conservative. For example, consider the following code:

$$
\text { int } \mathrm{x}=\operatorname{obj} \cdot \operatorname{get} \mathrm{X}() \text {; int } \mathrm{Y}=\operatorname{obj} \cdot \operatorname{get} \mathrm{Y}() \text {; }
$$

The variable obj indicates an object of the class defining the methods getX () and getY (). In this case, the
CFG/PDG constructor produces a data dependence from the first statement to the second statement since the value of the variable obj is both read and written in each statement. Precisely, this data dependence is dispensable only if the execution of the method getx ( ) never changes the state of the object obj (e.g., the method does not change the value of every field but only returns its value).

\subsection{Discussion}

Each of the former two tools was completed with little time and effort and comprised small amount of description (about 46 LOC and 52 LOC, respectively) because we were able to use an existing XSL processor and wrote code in a standardized and popular language without learning proprietary programming interfaces. The CFG/PDG constructor demonstrates that Sapid/XML provides sufficient information about source code, which is not inferior to that provided by the AST. Moreover, we confirmed that extending the original XSDML representation is useful for sharing and exchanging analyzed information. In fact, one new attribute was added in order to indicate locations of code fragments when we developed the cross reference extractor, and one new tag and one new attribute were used in the refactoring browser in order to express the changes of source code.

In addition to these tools, a tool allows developers to annotate any code fragment by using individual elements or attributes. For example, a version control tool might desire to attach information about the modified time to not only each file but also each method as follows:

$<$ Method modified="Mon Apr 5 10:45:14" > .

$</$ Method $>$

or it might assign an access permission for each method as follows:

$<$ Method mode="Read-only" $>$. </Method $>$

Additionally, developers might want to embed a temporary note that differs from a permanent comment into code.

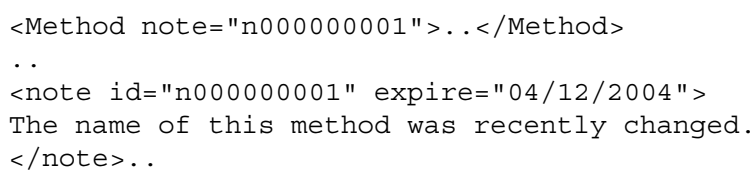

In this case, the unparser must be slightly modified and a proper editor (or viewer) displaying the textual contents of the added tag is needed to prepare.

\section{Experimental Results}

The XML representation of source code in general causes expansion of the file size and processing time because of its portability and flexibility. To roughly evaluate performance of Sapid/XML, we carried out simple experiments with four programs (Notepad, Stylepad, 
Table 2. Size of converted XSDML documents.

\begin{tabular}{|l||c|c|c||cc|c|c|}
\hline \multirow{2}{*}{ Program } & \multicolumn{3}{|c||}{ Java source file (.java) } & \multicolumn{4}{c|}{ XSDML file (.xml) } \\
\cline { 2 - 8 } & \# of files & LOC & Size [bytes] & .java.xml [bytes] & ratio & .class.xml [bytes] & Total [bytes] \\
\hline \hline Notepad & 2 & 1,343 & 38,805 & 412,522 & 10.63 & $1,239,167$ & $1,651,689$ \\
Stylepad & 5 & 2,159 & 65,245 & 717,249 & 11.00 & $1,433,866$ & $2,151,115$ \\
SwingSet2 & 31 & 8,617 & 294,619 & $3,088,867$ & 10.48 & $2,193,784$ & $5,282,651$ \\
Java2D & 62 & 14,187 & 509,949 & $6,355,034$ & 12.46 & $2,217,521$ & $8,572,555$ \\
\hline
\end{tabular}

Table 3. Processing time for conversion and manipulation of XSDML documents.

\begin{tabular}{|c|c|c|c|c|c|c|c|c|c|c|}
\hline \multirow{2}{*}{ Program } & \multicolumn{2}{|c|}{ XML file (.xml) } & \multicolumn{4}{|c|}{ Conversion time [s] } & \multicolumn{4}{|c|}{ Manipulation time [s] } \\
\hline & \# of files & \# of elements & Syntactic & Semantic & Total & Each file & Counter & Each file & Viewer & Each file \\
\hline$\overline{\text { Note }}$ & $\overline{2}$ & $\overline{20,634}$ & $\overline{5.444}$ & 22.077 & 27.521 & $\overline{13.761}$ & 0.032 & "0.016 & 2.250 & 1.125 \\
\hline Style & 5 & 35,418 & 9.336 & 28.793 & 38.129 & 7.626 & 0.034 & 0.007 & 4.970 & 0.994 \\
\hline SwingSet2 & 31 & 150,086 & 59.455 & 140.051 & 199.506 & 6.436 & 0.068 & 0.002 & 27.220 & 0.878 \\
\hline Java2D & 62 & 308,631 & 88.526 & 480.403 & 568.929 & 9.176 & 0.129 & 0.002 & 54.940 & 0.886 \\
\hline
\end{tabular}

SwingSet2, and Java2D) packaged in the Sun Microsystems J2SDK1.4.2.

Table 2 shows the size of the original Java source files and their converted XSDML files. The size of the converted XML files (.java.xml) is about 10 times $(10.63$, $11.00,10.48$, and 12.46 times, respectively) larger than that of original files. This is because our proposed XML representation contains various kinds of analyzed information about the source code. Moreover, Sapid/XML automatically generates summary XSDML documents (.class.xml) from classes related to Java source files. These files consume much space although they can be shared by respective programs. The repository size might cause the "out of memory" problem when Sapid/XML handles a huge program.

Table 3 shows two types of processing time. The conversion time denotes how long does it take to convert Java source files into XSDML documents. This time is divided into two phases: syntactic parsing and semantic analysis. The manipulation time was measured by using two applications. The "Counter" application traverses all elements (tags and attributes) and counts their numbers, which uses the DOM processor, Xerces2 Java Parser 2.6.2 [13]. The "Viewer" application generates a browsable source code in HTML form. It uses the XSL processor, Xalan Java version 2.6.0 [12] and the stylesheet described in Appendix A. The execution was performed on a computer with a Pentium4 $2.4 \mathrm{GHz}$ CPU and a 640MB of RAM, running Red Hat Linux9 and Sun Microsystems J2RE1.4.2 _01.

The conversion time for each Java source file is about 6 to 14 seconds and is much longer than the general compile time. This main reason is that Sapid/XML uses XSDML documents and an XML processor when performing global semantic analysis. This result might not be critical to build an application which seldom needs the conversion (e.g., a source code viewer or a software metrics tool) but it might be problematic to build interactive tools which need the frequent reconversion. To reduce the conversion time, we are planning to adopt the semantic analyzer of sophisticated compilers or modifying existing IDEs to generate XSDML documents. The manipulation time is considered as reasonable, which is about 1 second for each file.

\section{Conclusion}

Tool developers require more extensible and portable representations of tool platforms. This paper has proposed the XSDML representation using XML and Sapid/XML that is a tool platform for managing such representation. Sapid/XML retains original code fragments in the converted XSDML documents and inserts the globally analyzed information into them. With this platform, the developers easily build software tools that collaborate with each other.

For the platform to be truly practical, its performance must be improved and the development of many tools are needed. From functional points of view, Sapid/XML cannot replace an existing powerful IDE. Additionally, our proposed XSDML representation is not perfect and should be refined. We are planning to integrate the XSDML representation and its converter into popular IDEs (e.g., Eclipse [3]).

The Sapid/XML tool platform and some tools running on it can be downloaded from http://www.jtool.org.

\section{Acknowledgments}

The authors would like to thank Akinori Yonezawa, Etsuya Shibayama, Kiyoshi Agusa, and all members who have been engaging the Sapid project. We also thank the members of the ISR at the University of California, Irvine, who give us helpful and valuable comments. Finally, we thank Christopher Van der Westhuizen, Ping H. Chen, and the anonymous reviewers for their excellent suggestions 
that improve this paper. This work was sponsored by the Information-technology Promotion Agency (IPA), Japan.

\section{References}

[1] Byte Code Engineering Library (BCEL). http: //jakarta.apache.org/bcel/.

[2] Document Object Model (DOM). http: //www.w3 .org/DOM/.

[3] Eclipse. http://www.eclipse.org/.

[4] Extensible Markup Language (XML). http: //www.w3 .org/XML/.

[5] Extensible Stylesheet Language Family (XSL). http: //www.w3.org/Style/XSL/.

[6] GXL: Graph eXchange Language. http: //www . gupro.de/GXL/.

[7] JDOM. http://www.jdom.org/.

[8] Mozilla. http://www.mozilla.org/.

[9] RECORDER. http://recoder. sourceforge.net/.

[10] Semantic Designs, Inc., DMS Software Reengineering Toolkit. http: / / www. semdesigns. com/ Products/DMS/DMSToolkit.html.

[11] Simple API for XML (SAX). http: //www. saxproject.org/.

[12] Xalan-Java. http://xml. apache.org/xalan-j/.

[13] Xerces2 Java Parser. http: //xml apache.org/xerces $2-j /$.

[14] A. V. Aho, R. Sethi, and J. D. Ullman. Compilers: Principles, Techniques, and Tools. Addison-Wesley, 1986.

[15] G. J. Badros. JavaML: A markup language for Java source code. In Proc. Int'l WWW Conference, May 2000. http: //www9.org/w9cdrom/index.html.

[16] T. Ball and S. B. Horwitz. Slicing programs with arbitrary control flow. In Proc. Intl. Work. on Automated and Algorithmic Debugging, LNCS 749, pages 206-222, May 1993.

[17] M. Boshernitsan and S. L. Graham. Designing an XMLbased exchange format for Harmonia. In Proc. WCRE'00, pages 287-289, Nov. 2000.

[18] J. R. Cordy. Generalized selective XML markup of source code using agile parsing. In Proc. IWPC'03, pages 144-153, May 2003.

[19] J. Ebert, B. Kullbach, and A. Winter. GraX - An interchange format for reengineering tools. In Proc. WCRE'99, pages 89-98, Oct. 19991.

[20] J. Ferrante, K. J. Ottenstein, and J. D. Warren. The program dependence graph and its use in optimization. $A C M$ TOPLAS, 9(3):319-349, July 1987.

[21] J. Gosling, B. Joy, and G. Steele. The Java Language Specification. Addison-Wesley, 1996.

[22] M. J. Harrold, J. A. Jones, T. Li, D. Liang, A. Orso, M. Pennings, S. Sinha, S. A. Spoon, and A. Gujarathi. Regression test selection for Java software. In Proc. OOPSLA, pages 312-326, Oct. 2001.

[23] R. C. Holt, A. Winter, and A. Schürr. GXL: Toward a standard exchange format. In Proc. WCRE'00, pages 162-171, Nov. 2000.

[24] S. Horwitz, T. Ball, and D. Binkley. Interprocedural slicing using dependence graphs. ACM TOPLAS, 12(1):26-60, Jan. 1990.
[25] J. I. Maletic, M. Collard, and H. Kagdi. Leveraging XML technologies in developing program analysis tools. In Proc. Adoption-Centric Software Engineering (ACSE), pages 8085, May 2004.

[26] J. I. Maletic, M. L. Collard, and A. Marcus. Source code files as structured documents. In Proc. IWPC'02, pages 289-292, June 2002.

[27] E. Mamas and K. Kontogiannis. Towards portable source code representations using XML. In Proc. WCRE'00, pages 172-182, Nov. 2000.

[28] G. McArthur, J. Mylopoulos, and S. K. K. Ng. An extensible tool for source code representation using XML. In Proc. WCRE'02, pages 199-208, Oct. 2002.

[29] J. F. Power and B. A. Malloy. Program annotation in XML: a parse-tree based approach. In Proc. WCRE'02, pages 190 198, Oct. 2002.

[30] M. Weiser. Program slicing. IEEE Trans. Software Engineering (TSE), 10(4):352-357, July 1984.

\section{A. XSL Stylesheets (htmlview.xsl)}

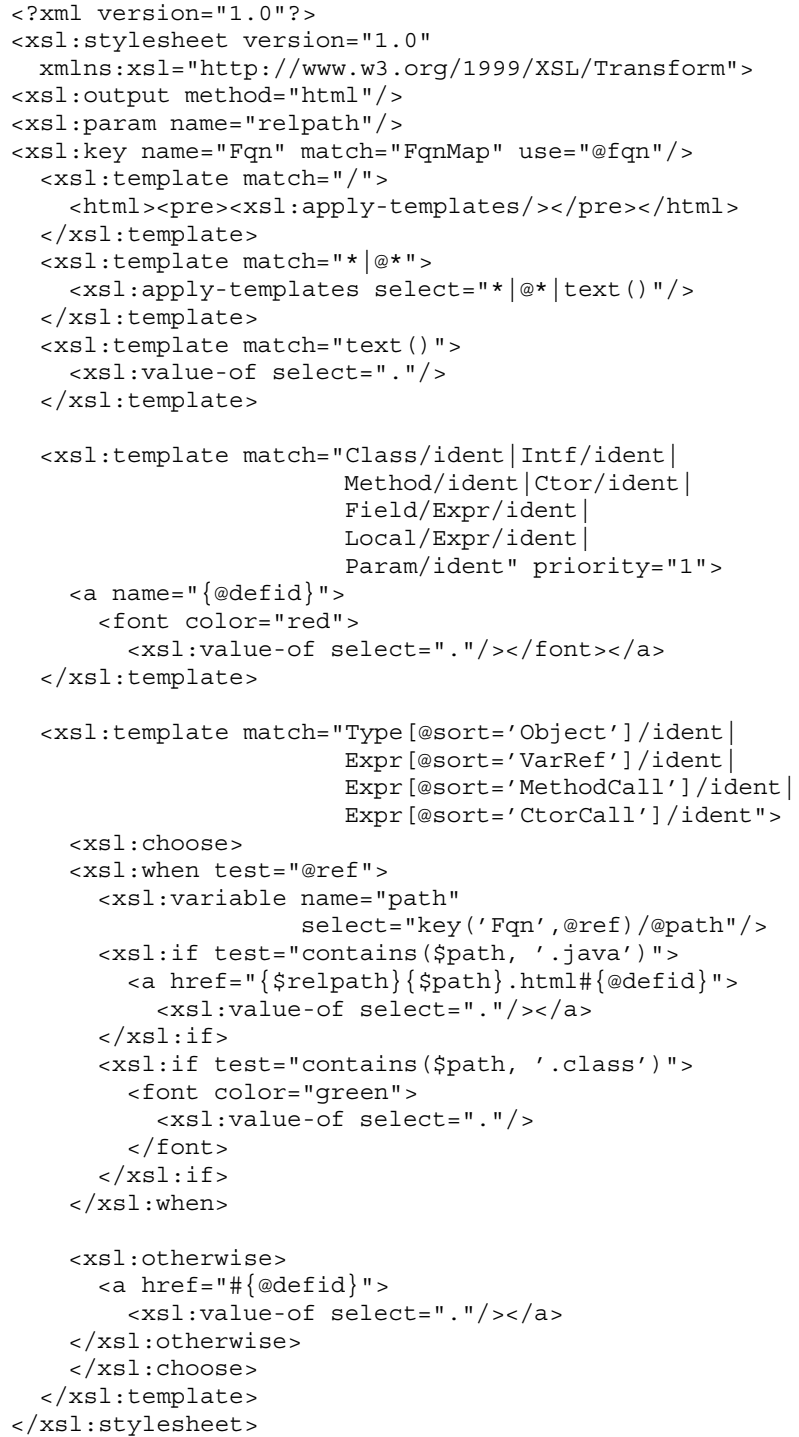

